\title{
La rehabilitación del suelo industrial. Herramientas de ordenación y gestión en la Comunidad Valenciana.
}

\author{
Carlos Esteve Aguado ${ }^{1}$, Juan Jaime Cano Hurtado², \\ Adrián García Martínez ${ }^{3}$, Ana Isabel Escoms Martínez ${ }^{4}$
}

${ }^{14}$ Departamento de Urbanismo, ${ }^{23}$ Departamento de Ingeniería de la Construcción y de Proyectos de Ingeniería Civil. Universidad Politécnica de Valencia, Valencia, España

E-mail: ${ }^{1}$ carlosesteve3@gmail.com, 2jjcano@cst.upv.es,

3adriangarma@gmail.com, ${ }^{4} 06349 @$ ctav.es

\begin{abstract}
Resumen. En la actualidad la dinámica de las ciudades ha provocado un importante giro hacia la rehabilitación urbana en todos sus aspectos. Desde la aprobación de la conocida como Ley de las $3 R$ (Ley de Regeneración, Rehabilitación y Renovación Urbana) y su posterior incorporación al Texto Refundido de la Ley del Suelo y Rehabilitación Urbana, los procesos urbanísticos en las ciudades están pasando de modelos centrados en la expansión hacia modelos centrados en la rehabilitación del suelo urbano consolidado.

La aplicación de los instrumentos de ordenación y gestión de actuaciones urbanisticas de rehabilitación de suelo urbano está enfocada hacia el uso residencial, tanto en la legislación estatal como en la autonómica en el caso de la Comunidad Valenciana. Las particularidades del suelo industrial consolidado hacen que la normativa resulte de difícil aplicación al suelo con este uso predominante.

Por otro lado, en la Comunidad Valenciana se ha aprobado recientemente la Ley de Gestión, Modernización y Promoción de Áreas Industriales de la Comunidad Valenciana que establece en esencia mecanismos para valorar la calidad de las áreas industriales e incorpora las entidades de gestión de áreas industriales, las cuales funcionarán de forma parecida a las comunidades de propietarios establecidas en la Ley de Propiedad Horizontal.

Se pondrá especial atención a la relación existente entre las herramientas que ofrecen las leyes de suelo y urbanisticas y la reciente ley sobre áreas industriales de la Comunidad Valenciana, tratando de establecer los aspectos que, dada su falta de definición, se deberían establecer en el desarrollo reglamentario de dicha Ley de manera que los requisitos establecidos en la misma lleguen a tener carácter de estándares urbanísticos.
\end{abstract}

Palabras clave: rehabilitación urbana, actividad económica,

suelo industrial, gestión urbanistica, áreas industriales.

\section{Introducción}

La Estrategia Territorial de la Comunitat Valenciana (en adelante ETCV) es el documento normativo básico para la planificación urbanística en la Comunitat Valenciana. Establece diferentes objetivos que afectan al suelo industrial en diferentes facetas. Por un lado, el objetivo 24 de la ETCV establece que se disponga de suelo destinado a actividades económicas en cantidad suficiente y adecuada. Por otro lado, el objetivo 16 de la ETCV persigue el convertir a la Comunitat Valenciana en la principal plataforma logística del Mediterráneo (Muñoz Criado y Doménech Gregori, 2011b, 2011a)

A su vez, la estrategia de política industrial de la Comunitat Valenciana (Generalitat 
Valenciana, 2014) establece como algunos de sus ejes más determinantes el fomento del crecimiento industrial, la diversificación y la modernización de la estructura productiva de la región.

Con el objetivo de coordinarse con las disposiciones anteriores en cuanto a la existencia de suelo industrial donde se pueda aprovechar la posición geográfica estratégica $\mathrm{y}$ las infraestructuras presentes y futuras del territorio, para incrementar la productividad y competitividad de los sectores económicos y para atraer nuevas empresas, se ha redactado un texto legislativo, novedoso por ser el primero con ese carácter, que persigue la modernización, la gestión y la promoción de las áreas industriales.

En el presente texto se van a analizar las deferentes herramientas e instrumentos de que se dispone para la rehabilitación del suelo industrial. Las actuaciones en el suelo urbano de carácter industrial suelen abordarse principalmente como proyectos urbanos, es decir, actuaciones separadas entre sí que persiguen dar solución a un problema concreto. Comúnmente nos podemos encontrar con actuaciones de reurbanización de las infraestructuras de un área industrial que nada tienen que ver con el resto de las preexistentes en la misma área industrial al no disponer de una planificación previa. Las posibilidades de regeneración integral de las áreas industrial y su necesidad de planificación dependerán de su estado inicial, debiendo confiar en un correcto análisis y diagnóstico previo para establecer las líneas de actuación más oportunas, como veremos a lo largo del texto.

Las claves para la modernización del suelo industrial a raíz de las nuevas disposiciones legislativas de la Comunidad Valenciana.

El mapa de suelo industrial de la Comunidad Valenciana, las Entidades de Gestión y Modernización de Áreas Industriales (en adelante EGM) y la calificación de las áreas industriales son las principales novedades que introduce la Ley de Gestión, Modernización y Promoción de Áreas Industriales de la Comunidad Valenciana (en adelante LGMPAICV). Con estas herramientas se persiguen los objetivos de convertir las áreas industriales existentes en espacios productivos de calidad, modernos y competitivos, que favorezcan la productividad y la competitividad de sus ocupantes.

\section{El mapa del suelo industrial de la Comunidad Valenciana.}

Con el fin de dar un impulso a la implantación de nuevas empresas en el suelo industrial existente en la Comunitat Valenciana se crea el mapa de suelo industrial disponible en la misma. La información ofertada por la citada herramienta es la siguiente:

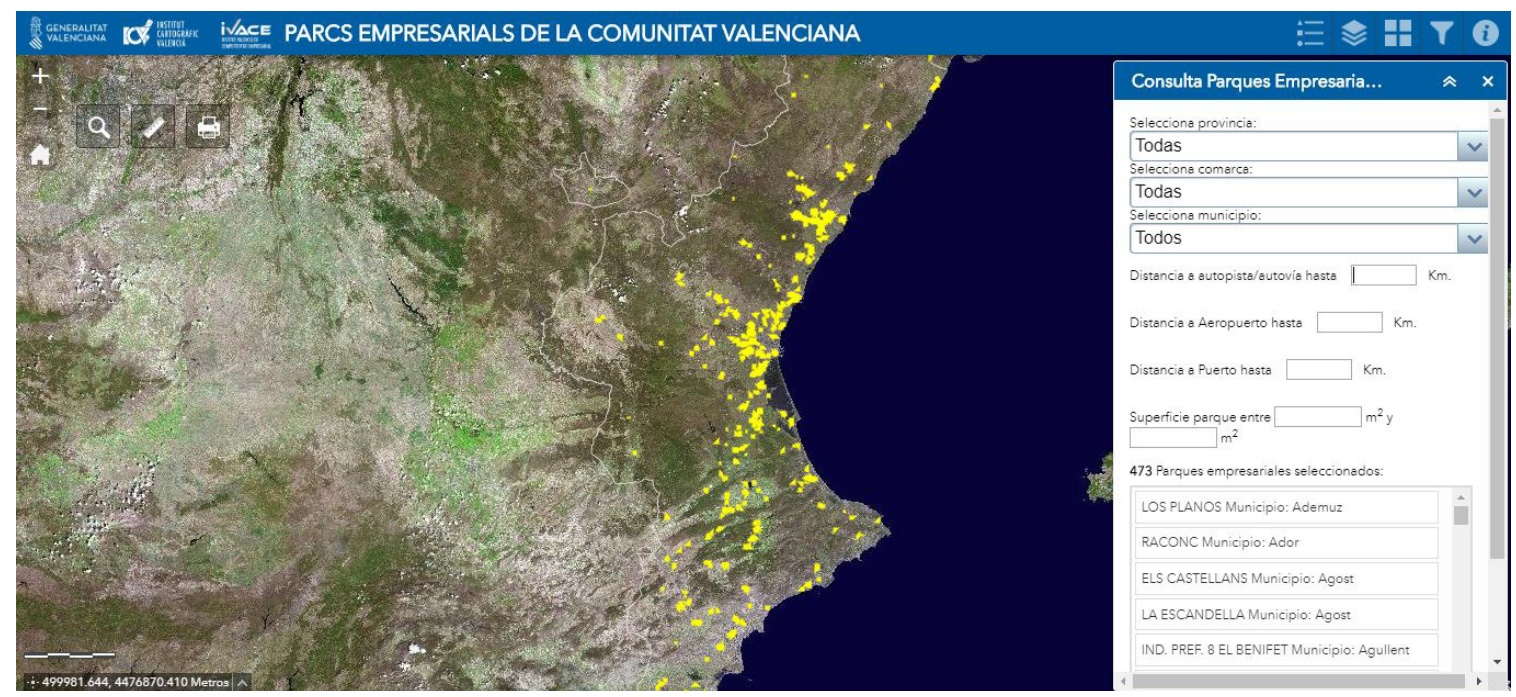

Fig. 1. Vista del visor del mapa de suelo industrial de la Comunitat Valenciana. 
-Situación de las áreas industriales.

-Descripción de los servicios disponibles en el área.

-La relación de cada área industrial con las principales infraestructuras viarias, ferroviarias y portuarias que permita ver su imbricación en las principales redes logísticas (red transeuropea de infraestructuras, corredor mediterráneo).

- Forma de gestión de cada área industrial.

Se ha configurado como un visor en línea, disponible a través de la página web del Instituto Valenciano de Competitividad Empresarial (en adelante IVACE), el cual permite realizar una búsqueda de áreas industriales relacionando dos conceptos. En primer lugar, su ubicación geográfica $\mathrm{y}$, en segundo lugar, una serie de inputs que el usuario puede establecer, como el rango de distancia a puertos y aeropuertos, la superficie total del área industrial, o la distancia a autopistas o autovías.

Se debe decir que la herramienta está desarrollada y es un buen punto de partida, pero la información que de cada área industrial se dispone es por el momento limitada debido a las dificultades de coordinación entre las distintas administraciones.

\section{Las entidades de gestión y modernización de las áreas industriales.}

Uno de los pilares básicos en la redacción de la LGMPAICV lo constituye la creación de la figura de las entidades de gestión y modernización de las áreas industriales. Se establecen sus disposiciones generales (naturaleza y régimen jurídico, integrantes, funciones, extremos básicos que deberán contener sus estatutos) y su régimen de organización y funcionamiento.

Las EGM estarán constituidas por los propietarios de suelo industrial del áreal. La ley no obliga a la creación de estas EGM sino que su creación se aprobará por la mayoría de los propietarios de suelo del área industrial. Sus funciones resultarán semejantes a las de las comunidades de propietarios que se establecen en los inmuebles constituidos en propiedad horizontal. No obstante, cabe destacar lo establecido en la Ley de Ordenación del Territorio, Urbanismo y Paisaje de la Comunidad Valenciana (en adelante LOTUP) en cuanto al mantenimiento de las obras de urbanización, que por lo general es responsabilidad de los ayuntamientos desde su recepción, careciendo de validez todo pacto o disposición que pretenda trasladar esa competencia a personas privadas, propietarios $o$ asociaciones de propietarios, sin colaboración y control público o sin condiciones o por tiempo indeterminado.

La colaboración de las EGM con los ayuntamientos respectivos se presume básica para la consecución de los fines de éstas, que no son otros que los de elevar los niveles de productividad y competitividad de las empresas integrantes del área. La participación de la sociedad (en este caso de los integrantes del área industrial) en los asuntos y decisiones públicas que les afecten quedará garantizada a través de estas entidades, estableciendo mecanismos que mejorarán la colaboración público-privada, primordialmente con la administración local, la cual es habitualmente la encargada del mantenimiento de dichas áreas industriales.

\section{Clasificación de los polígonos industriales.}

La LGMPAICV establece un sistema de clasificación de las áreas industriales de la Comunidad Valenciana según su nivel de dotaciones y de servicios ofertados. Concretamente las áreas industriales se podrán clasificar como básicas, consolidadas o avanzadas.

El nivel básico se obtiene siempre y cuando el área industrial esté clasificada y calificada como tal en el planeamiento urbanístico aplicable y además cumpla con los requisitos de la normativa urbanística y disponga de conexión a las redes de servicios. Quedarán fuera de esta clasificación por tanto aquellas áreas industriales cuyas empresas no dispongan de alguno de los servicios básicos que doten a las parcelas del polígono de la condición de solar. Recordemos que la condición de solar se obtiene cuando se dispone de abastecimiento de agua, de energía y de conexión a las redes de saneamiento, además de acceso rodado a la parcela. En definitiva, que no queden pendientes de ejecutar obras de urbanización. 
Para alcanzar el nivel consolidado, además de cumplir con las exigencias del nivel básico, el área industrial deberá tener al menos siete dotaciones de entre doce que establece la LGMPAICV, algunas de ellas de carácter obligatorio. Establece además para conseguir esta clasificación la obligatoriedad de haber constituido una EGM para el área industrial.

Por último, el nivel avanzado se alcanzará siempre que se disponga de nueve de las dotaciones establecidas para el nivel consolidado además de nueve dotaciones más de entre una lista de diecinueve que se ofrecen para el nivel avanzado.

El establecimiento de una clasificación para las áreas industriales que hace la LGMPAICV es un buen punto de partida para el establecimiento de mecanismos de rehabilitación de éstas, permitiendo saber que acciones se deben ir llevando a cabo para poder conseguir una clasificación superior a la inicial. No obstante, las dotaciones establecidas por la ley tienen carácter absoluto y se percibe que el simple cumplimiento de las mismas puede no garantizar unas áreas industriales de calidad.

Vemos por ejemplo que algunas de las dotaciones que la LGMPAICV establece son el disponer de oficinas bancarias o de establecimientos de restauración, sin establecer que proporción de dichos establecimientos puede resultar más adecuada. De esta manera, solo con tener uno de esos servicios un área industrial tendría esa dotación cubierta, y obviamente la calidad del área industrial no será la misma si su extensión es reducida que si es una gran área industrial.

Se detecta por tanto la necesidad de redacción de un reglamento que desarrolle la Ley y permita clasificar las áreas con mucho más rigor, estableciendo el nivel de dotación a que se deberá dar cumplimiento para su clasificación.

Estableciendo un paralelismo con los estándares urbanísticos, los cuales establece la LOTUP para el suelo de nueva urbanización, se deberán de establecer una serie de estándares dotacionales para las áreas industriales.
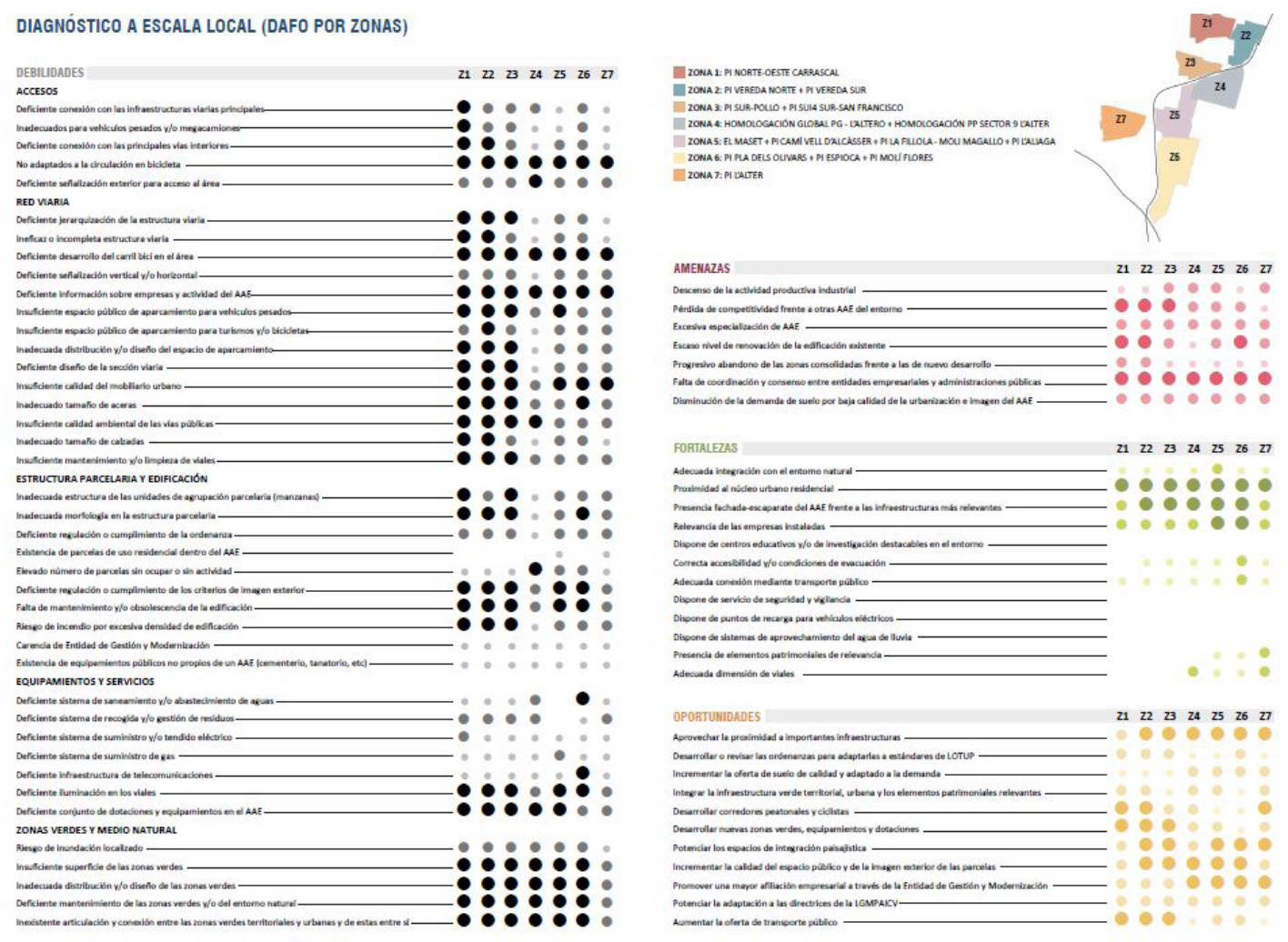

Fig. 2. Diagnóstico elaborado para el área industrial de Alcàsser - Beniparrell - Silla. 
Fig. 3. Plano de análisis que refleja la edad de la edificación del polígono industrial el Pla del municipio de Ontinyent, Valencia.

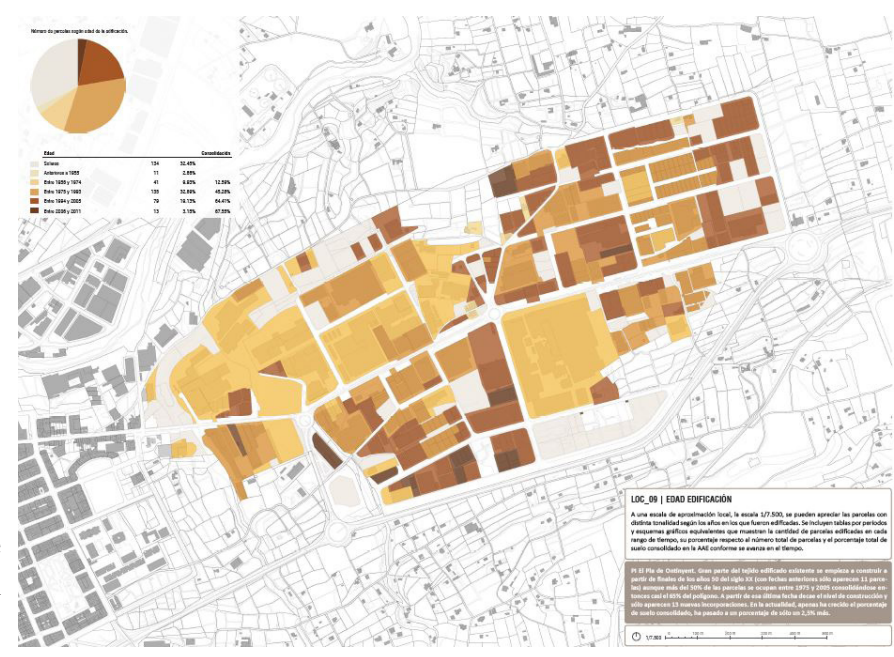

Instrumentos para la rehabilitación del suelo industrial existente.

La regeneración del suelo industrial necesitará de sus propios instrumentos de planificación y gestión para poder llevar a cabo las actuaciones en el medio y largo plazo, abordando su rehabilitación desde un punto de vista integral. Así pues, a las herramientas que la LGMPAICV nos ofrece habrá que añadir nuevos instrumentos de planificación urbanística y nuevos procesos de gestión que permitan rehabilitar los suelos que por el paso de los años han quedado obsoletos.(Cano Hurtado et al., 2017)(Cano Hurtado et al., 2017)

De los instrumentos de planificación establecidos por la LOTUP los planes de reforma interior permiten establecer nuevas ordenaciones urbanísticas para áreas concretas previamente determinadas en el plan general estructural. Estas áreas de reforma interior se deberán establecer en base a un análisis pormenorizado de cada área industrial que permita establecer un diagnóstico adecuado que se plasme en las propuestas de actuación concretas (Cano Hurtado 2018).

Establecer áreas de regeneración y renovación urbanas.

Si del análisis previo de las áreas industriales se diagnostica que en dicha área se deben efectuar reformas integrales la lógica lleva a pensar que estas se plasmen en un único documento. Las Áreas de Regeneración y Renovación Urbana (en adelante ARRU) son documentos que se vienen desarrollando para actuaciones en la ciudad consolidada pero siempre centrados en las áreas residenciales que tienen su problemática concreta, como centros históricos o áreas degradadas.(García Ponz, 2016)

Se deberá profundizar en la redacción de ARRU para áreas industriales, siendo esta lo solución idónea para áreas industriales que no lleguen a la clasificación de básica. Y es que si un área industrial no es ni siquiera básica ello quiere decir que tiene pendientes importantes obras de urbanización cuya ejecución deberá llevarse a cabo mediante actuaciones integradas.

\section{La gestión las áreas industriales consolidadas. La viabilidad económica de las actuaciones.}

Una vez se hayan desarrollado los documentos de planeamiento para la renovación del área, el ARRU o documento análogo que se establezca tiene la complicada misión de establecer de donde obtener la financiación para los proyectos de urbanización que se deriven de la misma. La lógica invita a pensar en una distribución de cargas entre los agentes implicados en el proceso que en definitiva serán las administraciones públicas y los propietarios de suelo. En suelo urbano consolidado suele resultar traumático para los propietarios de suelo el tener que abonar cuotas de urbanización, al entender éste que ya tiene todas sus cargas satisfechas. La clave estará por 


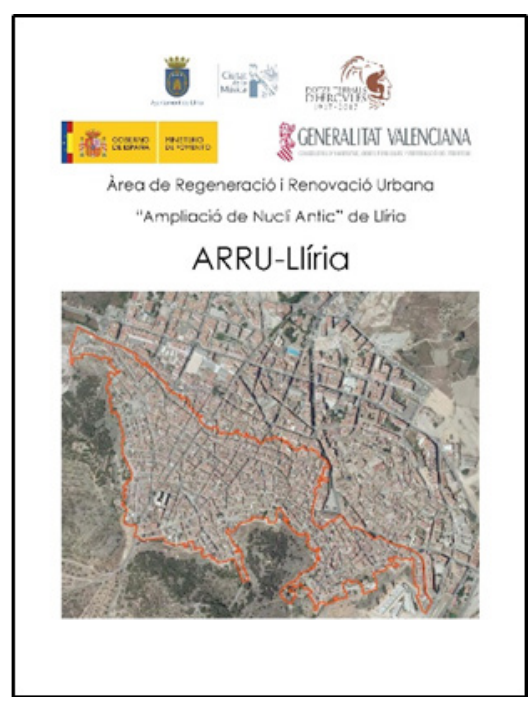

Fig. 4. Delimitación del área de regeneración y renovación urbana del municipio de Lliría, Valencia.

tanto en la memoria de viabilidad económica de la actuación, que en esencia viene a comparar el valor actual del suelo en cuestión con el valor futuro del mismo una vez se lleve a cabo la actuación, restándole el valor de las cuotas de urbanización que se deban satisfacer. (Fernández Fernández, Gerardo Roger. 2014)

Para que dicha viabilidad económica sea verdaderamente rentable para los propietarios de suelo se deberán valorar los cambios de uso localizados (de industrial a terciario e incluso a residencial en zonas con actividades compatibles) y el incremento de aprovechamiento que puede suponer para los usos industriales el incremento de la altura edificatoria (a más altura mayor capacidad de almacenaje). Todo ello pensando en la nueva imagen que se puede proyectar del área, regulando en un único documento nuevas alturas, usos entreplantas, retranqueos, vallados, condiciones estéticas, etc.

\section{Proyectos urbanos de modernización y mejora de infraestructuras y servicios.}

Las administraciones públicas tienden a realizar inversiones de carácter finalista cuando se trata del mantenimiento de las obras de urbanización. Esto provoca que muchas veces se lleven a cabo actuaciones aisladas que no son fruto de un análisis pormenorizado del área industrial en cuestión sino que se convierten en un mero parche.

Desde la administración regional se vienen destinando ayudas a entidades locales de carácter finalista, como es el caso de las "Ayudas para la modernización y dotación de infraestructuras y servicios en polígonos, áreas industriales y enclaves tecnológicos de la Comunitat Valenciana" del IVACE.

Dichas subvenciones se otorgan en concurrencia competitiva y entre las actuaciones subvencionables encontramos las de obra civil, instalaciones de vigilancia, implantación de transporte público, mejora en las instalaciones urbanas y mejora en los servicios del área.

Estas actuaciones pueden ser una gran opción para que áreas clasificadas como consolidadas puedan llegar a la clasificación de avanzadas y también para que áreas ya avanzadas aumenten la calidad de sus dotaciones y servicios.

\section{Conclusión.}

Se han descrito las principales herramientas de las que se dispone en la Comunitat Valenciana para la rehabilitación de áreas industriales. El debate se debe centrar en si esta regeneración se deberá llevar a cabo a través de programas concretos para fines concretos que produzcan

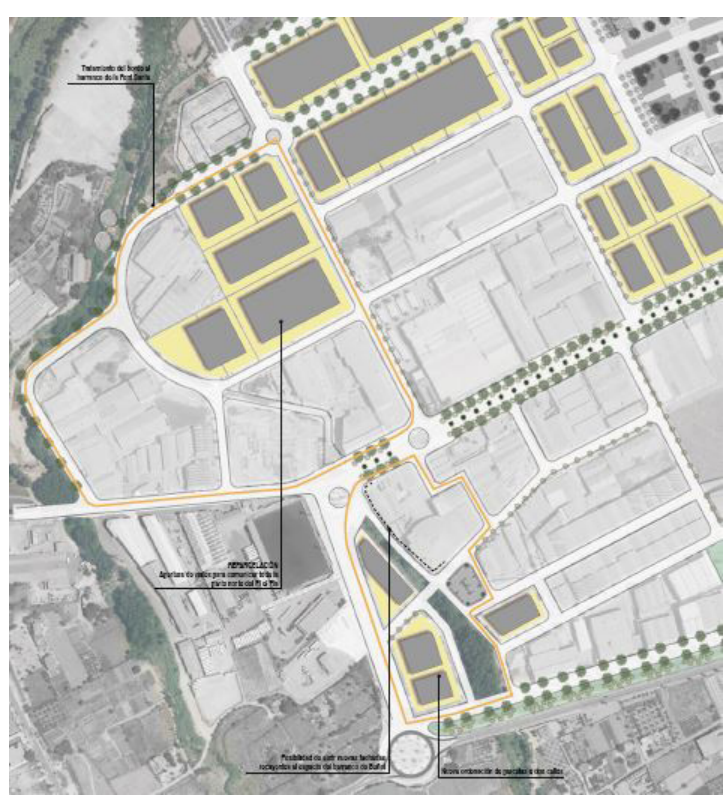

Fig. 5. Propuesta de ordenación para las áreas de regeneración y renovación urbanas propuestas para el polígono el Pla en Ontinyent, Valencia. 
que actuación a actuación, capa a capa, se vaya obteniendo un área industrial de mayor calidad o si estas actuaciones se deberán llevar a cabo de manera integral a través de planes específicos.

El análisis de la situación actual de cada área industrial y su diagnóstico arrojaran las necesidades específicas de cada una de ellas. En caso de que el área industrial solo necesite de pequeños ajustes e incrementos dotacionales en relación a las exigencias de clasificación de la LGMPAICV estas se podrán llevar a cabo mediante actuaciones aisladas. En caso de que el área en cuestión necesite de medidas integrales se deberán establecer protocolos de actuaciones urbanísticas análogos a las ARRU para áreas industriales. Las ayudas a proyectos concretos que se vienen realizando por parte de organismos públicos como el IVACE son un buen inicio y una buena solución para áreas que ya sean consolidadas pero la rehabilitación de las básicas solo se producirá cuando ésta sea integral.

El control de todo el proceso de rehabilitación industrial no podrá recaer en las Entidades de Gestión y Modernización de áreas industriales sin que a estas se les dote de más atribuciones que las que actualmente tienen por ley. Dado que la planificación urbanística es una potestad pública no susceptible de transacción, la coordinación de estas con las administraciones públicas será fundamental. Existe la posibilidad de que las EGM deriven hacia empresas públicas con un presupuesto que provenga tanto de capital público como de capital privado. De no ser así se corre el riesgo de que las EGM se constituyan en un mero grupo de presión en la defensa de sus intereses sin capacidad real de llevar a cabo iniciativas.

\section{Referencias}

Cano Hurtado, Juan Jaime. 2018. Renovación Urbana y Actividad Económica. Propuesta de Intervención Territorial En Un Enclave Logístico. Editorial Universitat Politècnica de València.

Cano Hurtado, Juan Jaime, María del Carmen Blasco Sánchez, Francisco Juan Martínez Pérez, Ana María Gascón Hernández, and Carlos Esteve Aguado. 2017. Renovación
Urbana. Áreas de Actividad Económica. La Modernización de Un Referente Industrial. Ontinyent. Editorial Universitat Politècnica de València.

— 2017 Renovación Urbana. Áreas de Actividad Económica. Las Oportunidades de Un Tejido Tradicional Junto a Grandes Infraestructuras. Editorial Universitat Politècnica de València.

Fernández Fernández, Gerardo Roger. 2014. Gestionar la ciudad consolidada: novedades instrumentales en la Ley Valenciana 5/2014 de Ordenación del Territorio, Urbanismo y Paisaje y en la Legislación Estatal, Texto Refundido de la Ley de Suelo 2/2008 y Ley 8/2013 de Rehabilitación, Regeneración y Renovación Urbanas. Valencia: Valencia: Tirant lo Blanch, 2014.

García Ponz, Albert. n.d. "Fórmulas Para La Rehabilitación, Regeneración y Renovación Urbanas En La Legislación Vigente." Edited by Luís Segura Gomis, Universitat Politècnica de València. Escuela Técnica Superior de Arquitectura - Escola Tècnica Superior d'Arquitectura, and Universitat Politècnica de València. Departamento de Urbanismo - Departament d'Urbanisme.

Institut Catalá del Sòl. 2007. Sectores de Actividad Económica. Libro de estilo. Barcelona: Institut Catalá del Sòl.

López Groh, Francisco. 2011. La regeneración de áreas industriales. Madrid: Sepes Entidad Estatal de Suelo.

Muñoz Criado, Arámzazu, and Vicente Doménech Gregori. 2011a. Actividad Económica.: Objetivo 24: Prever Suelo Para La Actividad Económica En Cantidad Suficiente y Adecuada. Valencia]: Valencia : Generalitat Valenciana. Conselleria de Medi Ambient, Aigua, Urbanisme i Habitatge, 2011.

2011b. Logística.: Objetivo 16: Convertir La Comunitat Valenciana En La Principal Plataforma Logística Del Mediterráneo. Valencia]: Valencia : Generalitat Valenciana. Conselleria de Medi Ambient, Aigua, Urbanisme i Habitatge, 2011.

Valenciana, Generalitat. 2014. Estrategia de politica industrial de la comunitat valenciana - Vision 2020. 